\title{
Desenvolvimento do programa de acreditação de organismos de certificação de sistemas de gestão de saúde e segurança ocupacional segundo a ISO 45001
}

\author{
Ricardo Kropf Santos Fermam \\ Engenheiro Químico, Mestre e Doutor em Tecnologia de Processos Químicos e Bioquímicos (UFRJ/EQ) \\ Gestor de Desenvolvimento de Programas de Acreditação (CGCRE/INMETRO) \\ Professor do Programa de Pós-graduação em Metrologia e Qualidade do INMETRO \\ 凶rkfermam@inmetro.gov.br \\ Daiana Leite Silva \\ Estudante de graduação em Engenheira Química (UFRJ/EQ). Estagiária (CGCRE/INMETRO) \\ 凶 dleitesilva@inmetro.gov.br \\ Andrea Barroso Melo Monteiro de Queiroz \\ Física (UFRJ), Mestre em Física (UFF) e Doutoranda em Física (UERJ) \\ Gerente da Divisão de Desenvolvimento de Programas de Acreditação (CGCRE/INMETRO) \\ Professora do Programa de Pós-graduação em Metrologia e Qualidade do INMETRO. \\ 凶amelo@inmetro.gov.br
}

\section{Resumo:}

Esse artigo tem como objetivos apresentar os conceitos envolvidos e discutir o desenvolvimento do programa de acreditação de organismos de certificação de sistemas de gestão de saúde e segurança ocupacional segundo a norma ISO 45001, pela Coordenação Geral de Acreditação do Inmetro. O método utilizado tem como base as diretrizes estabelecidas na norma internacional ISO/IEC 17011:2017. Os resultados referem-se aos requisitos aplicáveis para a acreditação desses organismos e na competência de auditores. Por fim, são apresentadas considerações no sentido da importância dessa atividade para o Brasil. Este artigo constitui-se numa referência basilar para organismos de acreditação no exterior que planejam lançar este programa, ou que planejam implementar uma área de desenvolvimento de novos programas de acreditação, quer pela escassez de publicações técnico-científicas na área quanto pela aplicação dos conceitos envolvidos.

Palavras-chave: ISO 45001, acreditação, saúde e segurança ocupacional, certificação, sistema de gestão. 


\title{
Development of the accreditation program for certification bodies of occupational health and safety management systems according to ISO 45001
}

\begin{abstract}
:
This article aims to present the concepts involved and discuss the development of the accreditation program for Occupational health and safety management systems, according to ISO 45001, by the General Coordination of Accreditation of Inmetro. The method is based on the guidelines established in the international standard ISO / IEC 17011: 2017. The results refer to the requirements applicable to the access of these bodies and the competence of auditors. Finally, considerations regarding the importance of this activity for Brazil are presented. This paper is a key reference for overseas accreditation bodies planning to launch this program, or planning to implement an area for the development of new accreditation programs, either by the scarcity of technical-scientific publications in the area or by the application of the concepts involved.
\end{abstract}

Keywords: ISO 45001, accreditation, occupational health and safety, certification, management system.

\section{Desarrollo del programa de acreditación de organismos de certificación de sistemas de gestión de salud y seguridad ocupacional según la ISO 45001}

\section{Resumen:}

Este artículo tiene como objetivos presentar los conceptos involucrados y discutir el desarrollo del programa de acreditación de organismos de certificación de sistemas de gestión de salud y seguridad ocupacional según la norma ISO 45001 por la Coordinación General de Acreditación del Inmetro. El método utilizado se basa en las directrices establecidas en la norma internacional ISO / IEC 17011: 2017. Los resultados se refieren a los requisitos aplicables para la acreditación de estos organismos y en la competencia de los auditores. Por último, consideraciones en el sentido de la importancia de esa actividad para Brasil son apresentadas. Este trabajo es una referencia clave para los organismos de acreditación en el extranjero que planean lanzar este programa o planean implementar un área para desarrollar nuevos programas de acreditación, ya sea por la escasez de publicaciones técnico-científicas en el área o por la aplicación de los conceptos involucrados.

Palabras-clave: ISO 45001, acreditación, salud and seguridade ocupacional, cetificación, sistema de gestión. 


\section{INTRODUÇÃo}

Segundo a Organização Internacional do Trabalho (OIT, 2018), existem mais de 2,78 milhões de mortes por ano em decorrência de acidentes de trabalho ou doenças relacionadas ao trabalho, além de 374 milhões de feridos e doenças não fatais. Além do enorme impacto sobre as famílias e comunidades, o custo para os negócios e economias é significativo: as estimativas da Organização das Nações Unidas (OIT, 2018) mostram que, em todo o mundo, o custo total de doenças, ferimentos e mortes representa 3,94\% do produto interno bruto (PIB) mundial, ou cerca de US\$2,99 bilhões, pelo custo direto e indireto de lesões e doenças (ISO, 2018; GASIOROWSKI-DENIS, 2018).

Conforme o levantamento realizado por GARNICA; BARRIGA (2018), no Brasil, ocorrem cerca de 720.000 acidentes de trabalho por ano, dos quais 2.800 são fatais, e implicam um custo de aproximadamente $2 \%$ do PIB. Estes acidentes implicam, na prática, em perdas de imagem e financeiras, diretas e indiretas, para as empresas envolvidas, implicando em última análise em perda de competitividade. Acidentes e doenças ocupacionais têm conseqüências adversas profundas; os trabalhadores são feridos, o equipamento é destruído, a quantidade e a qualidade da produção diminuem, há perdas econômicas devido à aposentadoria antecipada e ausência de funcionários, o que afeta adversamente a reputação e a competitividade da organização (MOHAMMADFAM, 2017).

Uma organização é responsável pela saúde ocupacional e segurança dos seus funcionários e de outros que podem afetar suas atividades. Esta responsabilidade inclui promover e proteger sua saúde física e mental (ISO 45001:2018). As questões de saúde e segurança devem ser consideradas em um estágio inicial das operações de uma organização para garantir a conformidade e o maior registro de segurança possível. Essas questões precisam ser identificadas, planejadas, organizadas, controladas, monitoradas e revisadas, de forma sistemática, na acepção do ciclo de Deming (também conhecido como ciclo PDCA ${ }^{1}$ ), mostrado esquematicamente na Figura 1. Daí a importância de que a organização possua um sistema de gestão que possibilite tal monitoramento.

\footnotetext{
${ }^{1}$ O Ciclo PDCA, ou Ciclo Plan-Do-Check-Act (Planejar, Executar, Verificar e Atuar) é usado para controlar e gerenciar a melhoria contínua, usado para planejamento e implementação de programas da qualidade.
} 
Figura 1 - Ciclo PDCA.

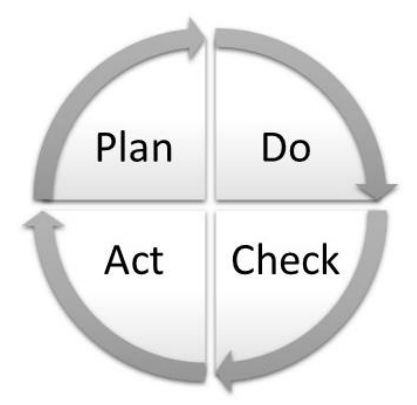

Fonte: Elaboração própria.

Em 2018, a International Organization for Standardization (ISO) publicou a norma ISO 45001- Sistemas de gestão de saúde e segurança no trabalho - Requisitos com orientação para uso, norma internacional que visa fornecer às organizações, independentemente do tamanho, do tipo e atividade, a estrutura para estabelecer, implementar, manter e melhorar um sistema de saúde e segurança ocupacional (SSO), eliminando perigos e minimizando riscos. A norma ISO 45001:2018 poderá substitutuir a norma OHSAS 18001 (BSI, 2007), padrão anteriormente usado.

Uma das principais diferenças entre a OSHAS 18001 (BSI, 2007) e a norma ISO 45001 (ISO, 2018) é o aumento da importância do envolvimento e participação dos trabalhadores, tornando a participação dos trabalhadores um alicerce fundamental na consecução do objetivo global de reduzir os ferimentos relacionados ao trabalho e a falta de saúde dos trabalhadores. Roncea (2018) enumera outras diferenças entre as referidas normas:

1) A ISO 45001 se concentra na interação entre uma organização e seu ambiente de negócios. Por sua vez, a OHSAS 18001 tem como foco o gerenciamento dos perigos ocupacionais para a saúde e segurança e outras questões internas;

2) A ISO 45001 é baseada em processos; já a OHSAS 18001 é baseada em procedimentos;

3) Diferentemente da OSHAS 18001, a ISO 45001 é dinâmica;

4) A ISO 45001 leva em consideração os riscos e oportunidades em saúde e segurança ocupacional (SSO), mas também outros riscos e oportunidades associados ao sistema de gerenciamento SSO; por seu turno, a OHSAS 18001 lida exclusivamente com riscos SSO; 
5) A ISO 45001 inclui requisitos das partes interessadas - OHSAS 18001 não.

6)

A intenção da Norma 45001 (ISO, 2018) é melhorar a segurança, a saúde e o bem-estar da força de trabalho e proporcionar um local de trabalho seguro tanto para a força de trabalho quanto para outras partes interessadas. Uma das formas de se comprovar o atendimento aos requisitos dessa norma é por meio de um processo de certificação acreditado, realizado por empresas independentes, imparciais e competentes para executarem esta atividade.

Esse artigo tem como objetivos apresentar os conceitos envolvidos e discutir o desenvolvimento do programa de acreditação de organismos de certificação de sistemas de gestão de saúde e seguirança ocupacional segundo a norma ISO 45001.

\section{REFERENCIAL TEÓRICO}

Um sistema de gestão (SG) é definido como sendo o conjunto de elementos interrelacionados ou interagentes de uma organização para estabelecer políticas, objetivos e processos de forma a alcançar esses objetivos. O SG inclui a estrutura da organização, papéis e responsabilidades, planejamento, operação, avaliação de performance e melhoria (ISO 45001:2018).

A intenção da adoção de um SG de saúde e segurança ocupacional (SGSSO) é prevenir lesões ou problemas de saúde de trabalhadores, quer sejam físicos, mentais ou cognitivos, e prover locais de trabalho seguros e saudáveis. Para isso, segundo Fonseca e Fermam (2015), um SGSSO estabelece uma estrutura que busca a melhoria contínua e, através de ações proativas, identifica, avalia e controla perigos e riscos existentes nos ambientes de trabalho, de modo que eles não se tornem causas de acidentes ou de doenças relacionadas ao trabalho e se mantenham dentro dos limites aceitáveis pelas partes interessadas.

O uso de sistemas de gestão da saúde e segurança ocupacional tem sido, durante muitos anos, a principal estratégia internacional para melhorar a saúde no trabalho (FRICK, 2011). A variedade de normas, diretrizes e auditorias de SGSSO desenvolvidas e disseminadas nos últimos 20 anos e o impacto desses sistemas na saúde e segurança dos funcionários, bem como respectivos os resultados econômicos associados foi estudada por Robson et al. (2007). De forma sintética, a análise dos trabalhos identificados pelos autores mostrou resultados 
geralmente positivos sobre a eficácia das intervenções do Sistema de Gestão de Saúde e Segurança do Trabalho (SGSST) ${ }^{2}$ na literatura publicada e revisada por pares, a despeito da baixa qualidade e quantidade de evidências identificadas.

Em seu estudo, PODGÓRSKI (2015) identificou como razões para o baixo nível de desempenho de SGSST o conhecimento e competência insuficientes dos auditores no domínio da SST e a ausência de verificação e validação das competências dos auditores. Esse empecilho é tradicionalmente superado por meio da sujeição dos organismos de certificação a um processo formal de acreditação.

A acreditação, definda mundialmente à luz da norma ISO/IEC 17000:2005 como sendo “atestação realizada por terceira parte relativa a um organismo de avaliação da conformidade (OAC), exprimindo demonstração formal de sua competência para realizar tarefas específicas de avaliação da conformidade", é um processo realizado com a finalidade de agregar confiança aos Organismos Avaliadores de Conformidade, como laboratórios e organismos certificadores ou de inspeção, reconhecendo sua competência. Tal atividade impacta em compradores, clientes e reguladores que estão envolvidos no produto, processo, serviço ou pessoas (SOARES e COSTA, 2006). A acreditação visa assegurar a coerência, competência técnica e a imparcialidade dos organismos que farão a certificação de sistemas de gestão de saúde e segurança ocupacional segundo a norma ISO 45001. Deste modo, as empresas que desejarem ter seus SGSSO certificados passam a contar com organismos de certificação previamente avaliados de forma independente contra normas reconhecidas internacionalmente.

No Brasil, a atividade de acreditação é atribuída ao Instituto Nacional de Metrologia, Qualidade e Tecnologia (Inmetro), o qual constitui-se no único organismo de acreditação, operando a norma internacional ISO/IEC 17011, reconhecido pelo Governo Federal. No âmbito desse Instituto, o planejamento, direção, orientação, coordenação e execução da função é realizada pela Coordenação Geral de Acreditação (CGCRE). A CGCRE atua, assim, como órgão acreditador de organismos de avaliação da conformidade e de outros organismos necessários ao desenvolvimento da infraestrutura de serviços tecnológicos no País, em conformidade com as normas, guias e regulamentos internacionalmente reconhecidos, sendo signatária de acordos internacionais de reconhecimento mútuo para suas atividades. De forma geral, a norma internacional aplicável na acreditação de organismos de certificação é

\footnotetext{
${ }^{2}$ Expressão sinonímia para Sistema de Gestão de Saúde e Segurança Ocupacional (SGSSO).
} 
a ISO/IEC 17021-1 "Conformity assessment - Requirements for bodies providing audit and certification of management systems - Part 1: Requirements" (ISO, 2015).

De forma simplicada, a atividade de aceditação de organismos de certificação envolve a realização de avaliações de escritório dos organismos demandantes e avaliações testemunhais. Conforme o DOQ CGCRE 015 (INMETRO, 2015), “estas avaliações são realizadas no local onde o organismo executa atividades correspondentes a uma auditoria, de forma completa, para determinar a extensão na qual os critérios de acreditação adotados pela CGCRE são atendidos. A testemunha da auditoria deve ser realizada para avaliar a seleção, qualificação, competência e monitoramento da equipe auditora designada pelo $\mathrm{OAC}$ e a implementação dos seus procedimentos de certificação de acordo com os critérios de acreditação adotados pela CGCRE."

Enquanto as avaliações de escritório tem como principal objetivo verificar o funcionamento do sistema de gestão interno desses organismos, as avaliações testemunhais objetivam verificar a competência da equipe auditora dos organismos e a implementação dos procedimentos inerentes aos processos de certificação durante a auditoria. Para isso, a equipe de avaliação da CGCRE reúne competência em duas áreas: em avaliação, cujo conhecimento abrange as normas e regulamentos relativos à acreditação, e em especialidade técnica, cujo conhecimento concentra-se na atividade da organização a ser auditada pelo organismo de certificação. Segundo o DOQ CGCRE 015 (INMETRO, 2015), o especialista "terá a função de avaliar se o especialista do organismo de certificação está familiarizado com os regulamentos legais aplicáveis; se possui conhecimento técnico apropriado das atividades específicas para as quais a certificação é desejada e, onde pertinente, com os procedimentos associados e o seu potencial de falha; se tem um grau de compreensão suficiente para realizar uma avaliação confiável da competência da organização para fornecer produtos, processos ou serviços, dentro do escopo da certificação".

Quando a CGCRE é demandada a realizar a acreditação para um programa específico, ainda não disponível em seu portfólio, procede-se a realização de estudos de viabilidade de ampliação da atividade de acreditação com vistas a desenvolver a infraestrutura interna necessária. Conforme explicam Fermam e Queiroz (2016), o processo de desenvolvimento requer a consideração da existência do programa de acreditação em outros países e como estão estruturados; a atualização ou criação de documentos internos; a elaboração de critérios de competência para a equipe de avaliação e, por fim, a seleção, o treinamento, a avaliação e a qualificação de avaliadores e especialistas técnicos para atuarem no programa. 


\section{MÉTODO}

A pesquisa é qualitativa e descritiva, realizada no âmbito da Coordenação Geral de Acreditação do INMETRO. Quanto aos meios, esta pesquisa classifica-se como sendo bibliográfica e documental. Relativamente à coleta de dados, trata-se de pesquisa-ação. A pesquisa-ação "caracteriza-se por uma interação efetiva e ampla entre pesquisadores e pesquisado. Seu objeto de estudo se constitui pela situação social e pelos problemas de naturezas diversas encontrados em tal situação" (LOPES, 2016). Baseado em Tripp (2005), é possível destacar algumas características de uma pesquisa-ação: (i) deve ser contínua, regularmente trabalhada para melhorar um aspecto dela; (ii) é pró-ativa com respeito à mudança, a qual é estratégica uma vez que a ação está baseada na compreensão alcançada por meio da análise de informações obtidas na pesquisa; (iii) a metodologia de pesquisa é subserviente à prática, possibilitando julgamentos baseados na melhor evidência passível de ser produzida; (iv) é participativa e colaborativa e (v) é sempre deliberativa.

O protocolo da pesquisa está baseado nas diretrizes estabelecidas na norma internacional ISO/IEC 17011:2017 "Conformity assessment - Requirements for accreditation bodies accrediting conformity assessment bodies", que especifica e padroniza a estrutura e o funcionamento dos organismos de acreditação mundialmente. Segundo essa norma, quando necessário desenvolver esquemas/programas de acreditação, o organismo de acreditação deve considerar: (i) a análise de sua competência atual, adequação da ampliação, recursos, dentre outros, no novo campo; (ii) o acesso e emprego de conhecimento especializado de outras fontes externas; (iii) a avaliação da necessidade de documentos de aplicação ou diretrizes; (iv) a seleção inicial e o treinamento de avaliadores e (v) o treinamento do pessoal no novo campo.

No Brasil, essas diretrizes foram incorporadas pela CGCRE por meio da norma NIE CGCRE 015 (INMETRO, 2015), a qual estabelece adicionalmente que no processo de desenvolvimento de um novo programa de acreditação devem ser considerados as diretrizes, recomendações, orientações e demais documentos de foros regionais e internacionais de acreditação e a existência de programa de avaliação da conformidade no país, fora do Brasil ou em nível internacional ou regional, o que significa, na prática, identificar se o programa de avaliação da conformidade para o qual solicita-se acreditação (o escopo de acreditação) é oferecido por algum organismo de acreditação congênere, no exterior (INMETRO, 2012). 
Desse modo, foi inicialmente realizada pesquisa nos sítios eletrônicos dos organismos de acreditação congêneres membros do Forum Internacional de Acreditação (IAF), com vistas a determinar quais implementam e operacionalizam o programa de acreditação para a norma ISO 45001, Sistema de Gestão de Saúde e Segurança Ocupacional. Foram identificados os seguintes organismos de acreditação: United Kingdom Accreditation Service (UKAS), Japan Accreditation Board (JAB), Entidad Nacional de Acreditación (ENAC) e American National Standards Institute - American Society for Quality National Accreditation Board LLC (ANAB).

Uma vez determinado quais organismos de acreditação possuíam o programa para a ISO 45001, pesquisou-se em seus documentos a forma como são especificados os escopos. Um escopo de acreditação é definido como sendo uma atividade específica de avaliação da conformidade para a qual a acreditação é procurada ou tem sido concedida (ISO, 2017). Na análise efetuada, constatou-se que os organismos de acreditação adotam o mesmo modelo de escopo, alinhado ao paradigma internacional estabelecido pelo Forum Internacional de Acreditação (International Accreditation Forum, IAF).

Além da análise junto aos organismos de acreditação, foi realizada uma pesquisa nos documentos mandatórios (MD) do IAF orientados para a ISO 45001. O IAF MD 22:2018 "Application of ISO/IEC 17021-1 for the Certification of Occupational Health and Safety Management Systems (OH\&SMS)" estabelece o escopo para Sistema de Gestão de Saúde e Segurança Ocupacional.

Para a avaliação testemunha, o IAF MD 22:2018 permite agrupar o escopo do código IAF por áreas técnicas ("technical clusters"). Nesses são agrupadas as atividades econômicas que pertencem ao mesmo segmento, facilitando a atividade de acreditação. Para cada cluster são especificados "códigos críticos" ("critical codes"). Um código crítico é um código que, de um ponto técnico, exige que a equipe de avaliação do organismo de certificação tenha um nível mais alto de: (i) competência (devido à complexidade dos processos / aspectos ambientais envolvidos), ou (ii) cautela (devido ao risco de não-conformidades e seu impacto, ou ao alto grau de regulação), ou (iii) diligência (devido aos comportamentos pessoais desejados que são importantes para o pessoal envolvido nas atividades de certificação, conforme necessário em um contexto específico). 


\section{RESULTADOS}

Identificou-se que os organismos de acreditação consultados operam a acreditação para a norma ISO 45001, Sistema de Gestão de Saúde e Segurança Ocupacional, de forma equivalente. Um critério importante que foi identificado, aplicado pelo UKAS, é a necessidade de que o avaliador do organismo de acreditação aplique adicionalmente a norma ISO/IEC TS 17021-10 - Conformity assessment - Requirements for bodies providing audit and certification of management systems - Part 10: Competence requirements for auditing and certification of occupational health and safety management systems (ISO/IEC, 2018) como parte do processo de avaliação, devendo a equipe de avaliação exigir evidências de que os requisitos desta especificação são levados em conta no processo de competência dos organismos de certificação. Diante da posição dos organismos de acreditação congêneres, confirmou-se a necessidade de incluir a ISO/IEC TS 17021-10 (ISO/IEC, 2018) como requisito a ser avaliado durante o processo de acreditação.

De forma geral, a ISO/IEC TS 17021-10 (ISO/IEC, 2018) expande as diretrizes para o pessoal envolvido no processo de certificação, conforme estabelecido na ISO/IEC 17021-1 (ISO/IEC, 2015). Ressalte-se que a norma ISO/IEC TS 17021-10 aplica-se diretamente ao pessoal envolvido na auditoria e na certificação de sistemas de gestão de saúde e segurança ocupacional, complementando os requisitos existentes na norma ISO/IEC 17021-1. Portanto, determinou-se que o organismo de certificação para a ISO 45001 deverá demonstrar o atendimento também aos requisitos dessa norma, além da própria ISO 45001 e ISO/IEC 170211 (acrescidos do IAF MD 21 e MD 22) durante o processo de acreditação, conforme ilustrado na Figura 2. 
Figura 2 - Normas aplicáveis na acreditação de organismos de certificação de sistemas de gestão de saúde e segurança ocupacional ISO 45001 (Programa de acreditação).

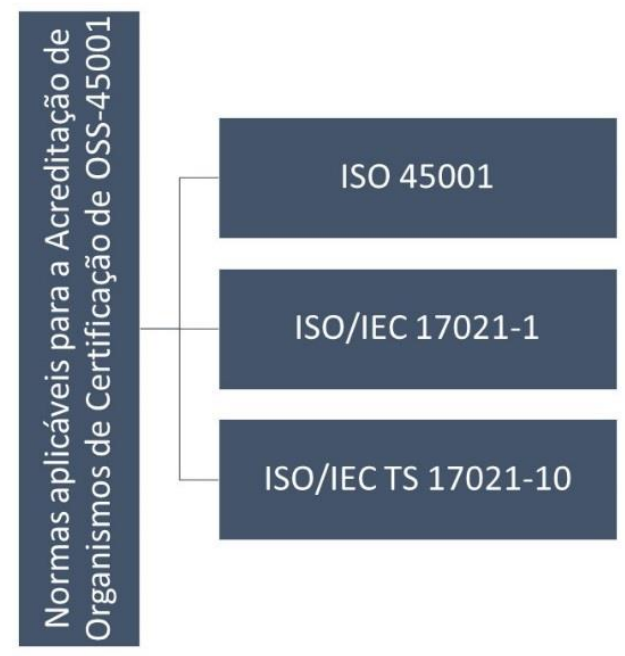

Fonte: elaboração própria.

A norma ISO/IEC TS 17021-10:2018 deixa claro que o papel do auditor do organismo de certificação não é o mesmo do inspetor do trabalho. Cada auditor de SGSSO deve ter conhecimento da sinergia desse sistema e como seus processos interagem de forma a propiciar um local de trabalho saudável e seguro, prevenindo acidentes e problemas de saúde. Além disso, a equipe auditora do organismo deve ter em seu conjunto o conhecimento (i) do papel e impacto da liderança e cultura sobre a organização, (ii) requisitos legais, relativos a saúde e segurança no trabalho bem como àqueles relacionados a área técnica específica de SSO; vale mencionar que o cumprimento dos requisitos legais é um dos fatores de sucesso ${ }^{3}$ para o SGSSO, segundo a norma ISO 45001, (iii) de riscos e oportunidades, de forma a avaliar se a organização aplica os métodos ${ }^{4}$ apropriados para identificar perigos ${ }^{5}$ e determinar riscos e oportunidades em SSO e outros riscos e oportunidades no contexto organizacional, (iv) de avaliação dos riscos em SSO, (v) dos tipos de processos que podem levar a uma melhoria na performance em SSO, (vi) das potenciais situações de emergência e suas respostas usuais, (vii)

\footnotetext{
${ }^{3}$ A adoção da norma ISO 45001 não garante, por si só, a prevenção de acidentes e doenças relacionadas ao trabalho para os trabalhadores, o fornecimento de locais de trabalho seguros e saudáveis e a melhoria do desempenho em SSO. É imprescindível que a organização obedeça a legislação aplicável.

${ }^{4}$ Exemplos de métodos relevantes e critérios para determinar riscos e oportunidades podem incluir análises qualitativas e quantitativas, como o Estudo de Perigo e Operabilidade (Hazard and Operability Studies, HAZOP) e a Análise de Modos de Falhas e Efeitos (Failure Mode and Effects Analysis, FMEA).

${ }^{5}$ Esses perigos incluem, mas não se limitam as seguintes categorias: físicos, químicos, biológicos, fisiológicos, mecânicos, elétricos, psicossociais e baseados em movimento e energia.
} 
em métodos de avaliação de performance proativos e reativos, incluindo indicadores qualitativos e quantitativos, (viii) em métodos ou medidas de controle usadas para eliminar perigos e reduzir riscos em SSO e (ix) em métodos de investigação de incidentes, tais como análise de causa-raiz e análise de árvore de falhas, de forma a avaliar se a organização conduz uma investigação eficientemente no seu contexto e no âmbito de seu SGSSO.

Diante da elucidação dos requisitos do programa de acreditação, foi determinada a competência de pessoal - avaliadores e especialistas - que farão a avaliação de acreditação nos organismos de certificação. Para os avaliadores, determinou-se que todos devam possuir experiência profissional compatível com a função de avaliação e curso de técnicas de avaliação/auditoria, além de cursos específicos nas normas específicas utilizadas na acreditação. Complementarmente, para este programa, determinou-se que os avaliadores deverão ter curso de interpretação da norma ISO 45001 e conhecimentos acerca da norma ISO/IEC TS 17021-10.

Quanto aos especialistas, após consultas a profissionais, individualmente, bem como aos conselhos e fundações que atuam na área de engenharia de segurança do trabalho, determinou-se que estes devam possuir nível superior em engenharia ou arquitetura; atuação na área de especialidade direta do serviço a ser avaliado e curso de especialização em Engenharia de Segurança e Saúde no Trabalho.

\section{CONSIDERAÇÕES FINAIS}

Muitos compradores exigem que seus fornecedores implementem um sistema de gestão como um pré-requisito para fazer negócios com eles. A certificação acreditada de sistemas de gestão baseados em normas técnicas, mecanismo utilizado na comprovação de que a empresa possui um sistema aderente a estes padrões, vem se tornando fundamental para a competitividade empresarial, possibilitando a melhoria contínua de suas operações. $O$ padrão de segurança e saúde ocupacional OHSAS 18001 ganhou considerável aceitação em todo o mundo, e firmas de diversos setores e de tamanhos variados o implementaram (FERNÁNDEZ-MUÑIZ, MONTES-PEÓN e VÁZQUEZ-ORDÁS, 2012).

Com o desenvolvimento do novo padrão internacional para sistemas de gestão de saúde e segurança ocupacional, ISO 45001, vários organismos de acreditação ao redor do 
mundo passaram a gerir programas de acreditação segundo esta norma. Atento a isso, o Fórum Internacional de Acreditação (IAF) estabeceu regras de forma a harmonizar as acreditações de organismos de certificação segundo a ISO 45001, de forma a evitar a criação de possíveis obstáculos comerciais baseados em diferentes esquemas de acreditação.

O desenvolvimento de um novo programa de acreditação pelo organismo de acreditação brasileiro é uma atividade que permite a contínua ampliação da oferta de serviços relevantes e atualizados para a sociedade e as empresas no país, tal desenvolvimento é estruturado de forma a considerar as regras e diretrizes estabelecidas nos foros internacionais e os programas já disponíveis noutros países, dito desenvolvimento possibilita que as acreditações realizadas no Brasil gozem de reconhecimento no exterior, reduzindo possíveis medidas protecionistas de base técnica e, consequentemente, favorecendo o acesso a mercados de produtos e serviços.

O artigo abordou as etapas, discussões e resultados do desenvolvimento do programa de acreditação para organismos de certificação de sistemas de gestão de saúde e seguirança ocupacional segundo a norma ISO 45001, no âmbito da Coordenação Geral de Acreditação do Inmetro. Ao longo do mesmo, mostrou-se os principais elementos de um programa de acreditação que seja capaz de agregar confiança as partes interessadas. Este programa encontra-se atualmente disponível para solicitação, por parte dos organismos de certificação interessados em ter sua competência técnica e imparcialidade reconhecidas para atuar na certificação de SGSSO segundo a ISO 45001.

\section{REFERÊNCIAS}

INMETRO, 2015a. DOQ CGCRE 015 - Orientação para participação de especialistas em testemunha das auditorias da CGCRE: documento de caráter orientativo. Disponível em: http://www.inmetro.gov.br/sidoq/arquivos/CGCRE/DOQ/DOQ-CGCRE-15_04.pdf. Acesso: fev. 2019.

INMETRO, 2015b. NIE-CGCRE-015 - Estudo de Viabilidade e Desenvolvimento de Novos Programas de Acreditação. Disponível em: http://intranet.inmetro.gov.br/Sidoq/arquivos/Cgcre/NIE/NIE-Cgcre-15_09.pdf. Acesso: jul. 2019. 
FERMAM, Ricardo Kropf Santos; DE QUEIROZ, Andrea Barroso Melo Monteiro. Development and Implementation of CGCRE Accreditation Program for Greenhouse Gas Verification Bodies. Journal of Physics: Conference Series. Vol. 733. No. 1. IOP Publishing, 2016.

FERNÁNDEZ-MUÑIZ, Beatriz; MONTES-PEÓN, José Manuel; VÁZQUEZ-ORDÁS, Camilo José. Safety climate in OHSAS 18001-certified organisations: Antecedents and consequences of safety behaviour. Accident Analysis \& Prevention, v. 45, p. 745-758, 2012.

FONSECA, Ivan Fagundes; FERMAM, Ricardo Kropf Santos. Sistema de Gestão de Segurança e Saúde no Trabalho: Uma Proposta de Avaliação da Conformidade para a Administração Pública Federal Brasileira. Sistemas \& Gestão, v. 10, n. 1, p. 16-28, 2015.

FRICK, Kaj. Worker influence on voluntary OHS management systems-A review of its ends and means. Safety Science, v. 49, n. 7, p. 974-987, 2011.

GARNICA, G. B., \& BARRIGA, G. D. C. (2018). Barriers to occupational health and safety management in small Brazilian enterprises. Production, 28, e20170046. http://dx.doi.org/10.1590/0103-6513.20170046.

GASIOROWSKI-DENIS, Elizabeth. Nuestro mundo con ISO 45001. In: Salud y Seguridade $\mathbf{n}$ el Trabajo: ¿Está preparado para ISO 45001? ISOfocus 127, Marzo-abril 2018. Disponível em: https://www.iso.org/files/live/sites/isoorg/files/news/magazine/ISOfocus\%20(2013NOW)/sp/ISOfocus_127_sp.pdf. Acesso: fev. 2019.

INTERNATIONAL ACCREDITATION FORUM. Application of ISO/IEC 17021-1 for the Certification of Occupational Health and Safety Management Systems (OH\&SMS). Issue 1. IAF MD 22:2018. IAF Mandatory Document. Disponível em: https://www.iaf.nu/workstation/upFiles/IAFMD22OHSMSPub25012018.pdf. Acesso: fev. 2019.

INMETRO (Instituto Nacional de Metrologia, Qualidade e Tecnologia) (2012) NIE-CGCRE-015: Estudo de Viabilidade e Desenvolvimento de Novos Programas de Acreditação. Rio de Janeiro: Inmetro. Disponível em: http://www.inmetro.gov.br/sidoq/arquivos/CGCRE/NIE/NIE-CGCRE-15_07.pdf. Acesso: fev. 2019.

INTERNATIONAL ORGANIZATION FOR STANDARDIZATION. Occupational health and safety ISO 45001. International Organization for Standardization. February 2018. Disponível em: https://www.iso.org/files/live/sites/isoorg/files/store/en/PUB100427.pdf. Acesso: 27 fev. 2019.

INTERNATIONAL ORGANIZATION FOR STANDARDIZATION/INTERNATIONAL ELECTROTECHNICAL COMMISSION. Conformity assessment - Vocabulary and general principles. 17000: 2005. Geneva: ISO/IEC, 2005.

INTERNATIONAL ORGANIZATION FOR STANDARDIZATION/INTERNATIONAL ELECTROTECHNICAL COMMISSION. Conformity assessment - Requirements for bodies providing audit and certification of management systems - Part 1: Requirements. 17021-1: 2015. Geneva: ISO/IEC, 2015 
Desenvolvimento do programa de acreditação de organismos de certificação de sistemas de gestão de saúde e segurança ocupacional segundo a ISO 45001

INTERNATIONAL ORGANIZATION FOR STANDARDIZATION/INTERNATIONAL ELECTROTECHNICAL COMMISSION. Conformity assessment - Requirements for accreditation bodies accrediting conformity assessment bodies: ISO/IEC 17011:2017. Geneva, 2017.

INTERNATIONAL ORGANIZATION FOR STANDARDIZATION (ISO). Occupational health and safety management systems - Requirements with guidance for use: ISO 45001:2018. Geneva, 2018.

INTERNATIONAL ORGANIZATION FOR STANDARDIZATION//INTERNATIONAL ELECTROTECHNICAL COMMISSION. Conformity assessment - Requirements for bodies providing audit and certification of management systems - Part 10: Competence requirements for auditing and certification of occupational health and safety management systems: ISO/IEC TS 17021-10:2018. Geneva, 2018.

LOPES, Jorge. Fazer Do Trabalho Científico Em Ciências Sociais Aplicadas, o. Editora Universitária UFPE, 2016.

MOHAMMADFAM, Iraj; KAMALINIA, Mojtaba; MOMENI, Masour; GOLMOHAMMADI, Rostan; HAMIDI, Yadollah; SOLTANIAN, Alizera. Evaluation of the quality of occupational health and safety management systems based on key performance indicators in certified organizations. Safety and health at work, v. 8, n. 2, p. 156161, 2017.

OSHAS 18001 Occupational health and safety management systems: Requirements. BSI, 2007.

OIT. Melhorar a Segurança e a Saúde dos Trabalhadores Jovens. Relatório. 2018. Disponível em: https://www.ilo.org/wcmsp5/groups/public/---ed_protect/---protrav/--safework/documents/publication/wcms_626351.pdf. Acesso: jul. 2019.

PODGÓRSKI, Daniel. Measuring operational performance of OSH management system-A demonstration of AHP-based selection of leading key performance indicators. Safety science, v. 73, p. 146-166, 2015.

ROBSON, Lynda S.; CLARKE, Judith A.; CULLEN, Kimberley; BIELECKY, Amber; SEVERIN, Colette; BIGELOW, Philip L.; IRVIN, Emma; CULYER, Anthony; MAHOOD, Quenby. The effectiveness of occupational health and safety management system interventions: a systematic review. Safety science, v. 45, n. 3, p. 329-353, 2007.

RONCEA, Cristian. Provocările migrării de la OHSAS 18001 la ISO 45001. CCF 2018, $26-28$ September 2018, Sinaia Romania. Disponível em: http://www.srac.ro/files/documente/Provocarile_migrarii_la_ISO_45001.pdf. Acesso: fev. 2019.

SOARES, M. A.; COSTA, H. G.: Competição na acreditação de laboratórios. XIII SIMPEP, Bauru, 2006.

TRIPP, David. Pesquisa-ação: uma introdução metodológica. Educação e pesquisa, v. 31, n. 3, p. 443-466, 2005.

\section{(cc) EY}

Este trabalho está licenciado com uma Licença Creative Commons - Atribuição 4.0 Internacional. 